\title{
OPEN Characteristics and outcomes of clinically diagnosed RT-PCR swab negative COVID-19: a retrospective cohort study
}

\author{
Paul Middleton ${ }^{1,6}$, Pablo N. Perez-Guzman $\mathbb{1}^{2,6}$, Alexandra Cheng ${ }^{1}$, Naveenta Kumar ${ }^{1}$, \\ Mara D. Kont ${ }^{2}$, Anna Daunt ${ }^{1}$, Sujit Mukherjee ${ }^{1}$, Graham Cooke ${ }^{1,2,3}$, Timothy B. Hallett ${ }^{2}$, \\ Katharina Hauck ${ }^{2,4}$, Peter J. White ${ }^{2,4,5}$, Mark R. Thursz ${ }^{1}$ \& Shevanthi Nayagam ${ }^{1,2 \bowtie}$
}

Patients with strong clinical features of COVID-19 with negative real time polymerase chain reaction (RT-PCR) SARS-CoV-2 testing are not currently included in official statistics. The scale, characteristics and clinical relevance of this group are not well described. We performed a retrospective cohort study in two large London hospitals to characterize the demographic, clinical, and hospitalization outcome characteristics of swab-negative clinical COVID-19 patients. We found 1 in 5 patients with a negative swab and clinical suspicion of COVID-19 received a clinical diagnosis of COVID-19 within clinical documentation, discharge summary or death certificate. We compared this group to a similar swab positive cohort and found similar demographic composition, symptomology and laboratory findings. Swab-negative clinical COVID-19 patients had better outcomes, with shorter length of hospital stay, reduced need for $>60 \%$ supplementary oxygen and reduced mortality. Patients with strong clinical features of COVID-19 that are swab-negative are a common clinical challenge. Health systems must recognize and plan for the management of swab-negative patients in their COVID-19 clinical management, infection control policies and epidemiological assessments.

As of 15th December 2020, the World Health Organization (WHO) has reported 70 million confirmed cases of COVID-19 globally with 1.6 million confirmed deaths ${ }^{1}$. WHO defines a confirmed case as a person with laboratory confirmation of COVID-19 infection ${ }^{2}$. Previously cases where laboratory confirmation is not done or inconclusive are recognized as probable cases however those with strong clinical features, but negative testing are not recognized ${ }^{2}$. Recent changes to WHO case definitions now allow probable cases to include patients who meet clinical and epidemiological criteria or patients with severe acute respiratory illness who have typical chest imaging features or unexplained anosmia or ageusia and do not stipulate the results of any performed laboratory testing ${ }^{3}$. Epidemiological evaluations in many countries however, including the UK, focus on patients with positive real-time polymerase chain reaction (RT-PCR) testing ${ }^{4}$. This places a significant importance on the diagnostic accuracy of laboratory testing.

However, the diagnostic accuracy of RT-PCR upper respiratory tract swabs is increasingly being questioned. A study utilizing both chest computerized tomography imaging (CT) and RT-PCR testing in patients with suspected COVID-19 found 75\% of cases with a negative RT-PCR test had CT findings suggestive of COVID-195. Several case reports describe patients with clinical features of COVID-19 but negative upper respiratory tract swabs who later have positive confirmatory testing on induced sputum or bronchial lavage $\mathrm{e}^{6-8}$. An analysis of published cohorts calculated the false-negative rate of RT-PCR testing amongst symptomatic COVID-19 patients who eventually test positive for COVID- 19 is likely $20 \%$ and increases gradually with time since symptoms onset ${ }^{9}$.

Acknowledging this, some patients with strong features of COVID-19 receive a clinical diagnosis of COVID19 despite a negative swab result. This clinical approach is being further recognized in the admission criteria of some clinical trials who permit recruitment of these patients ${ }^{10}$. For example in the recently reported RECOVERY trial $10 \%$ of those randomized to dexamethasone had a negative swab at the time of randomization ${ }^{11}$. However,

${ }^{1}$ Imperial College Healthcare NHS Trust, London, UK. ${ }^{2}$ MRC Centre for Global Infectious Disease Analysis, Imperial College London, London W2 1PG, UK. '3 Department of Infectious Diseases, Imperial College London, London, UK. ${ }^{4}$ NIHR Health Protection Research Unit in Modelling and Health Economics, Imperial College London, London, UK. ${ }^{5}$ Modelling and Economics Unit, National Infection Service, Public Health England, London, UK. "These authors contributed equally: Paul Middleton and Pablo N. Perez-Guzman. ${ }^{\square}$ email: s.nayagam01@imperial.ac.uk 


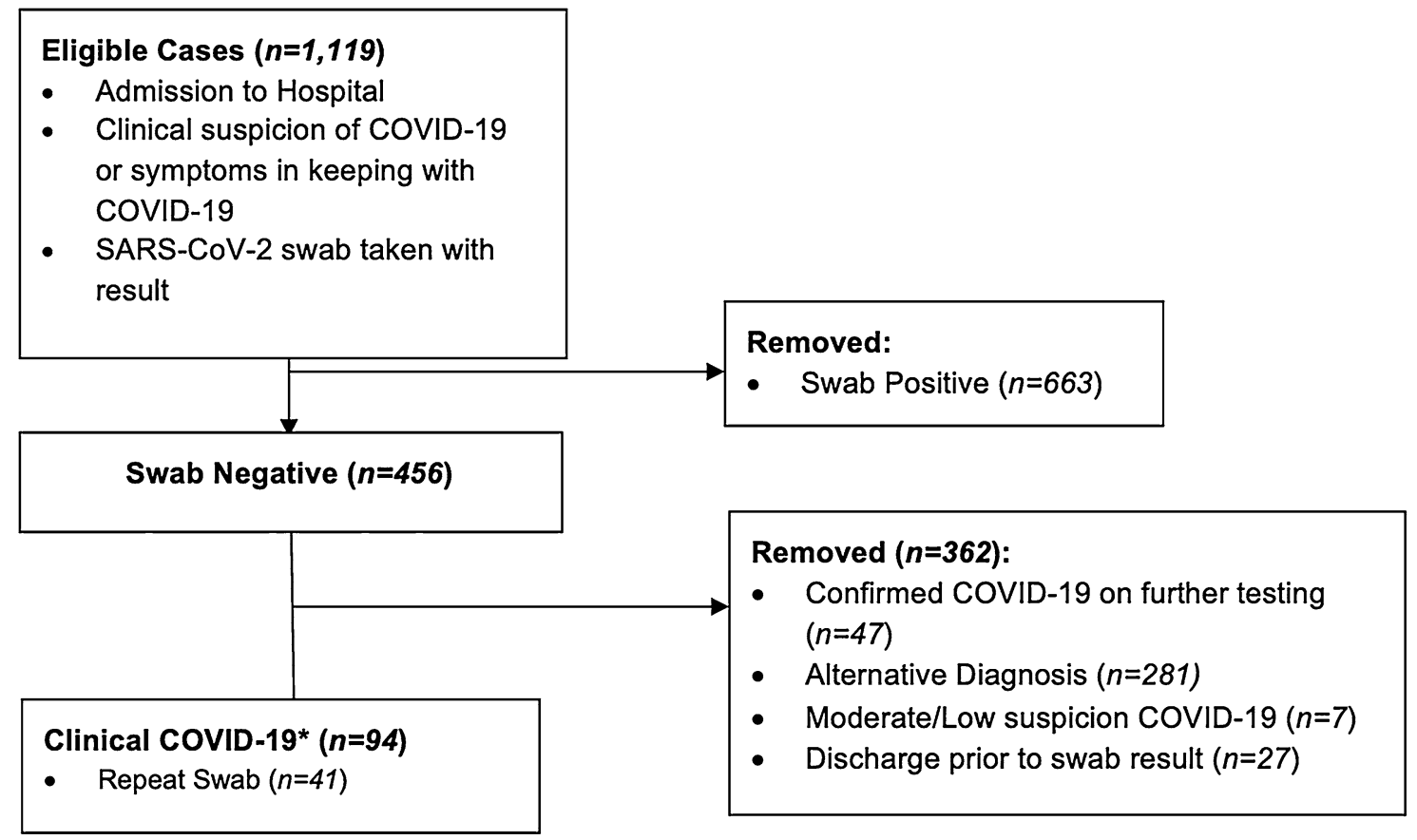

Figure 1. Case identification. ${ }^{\star} 98 \%(92 / 94)$ Clinical COVID-19 cases fulfilled PHE guidance on testing eligibility.

little has been documented about the scale and clinical relevance of this subgroup of patients for whom there is no consensus on optimal management. Our study aims to assess the real-world prevalence and characteristics of clinically diagnosed swab-negative COVID-19, including factors associated with swab-negative disease, and whether their outcomes differ to swab-positive patients.

\section{Methods}

We retrospectively reviewed medical admissions from March 1 to April 12, 2020 at Imperial College Healthcare Trust (ICHNT) in London, UK from two admitting sites. We defined eligible cases as those who presented with clinical suspicion of COVID-19 or had symptoms compatible with COVID-19, were admitted to hospital and had a SARS-CoV-2 nasopharyngeal swab performed. We collected full demographic characteristics, time course of symptoms, time of presentation and testing, presenting symptoms, final diagnosis and outcome as well as radiological and laboratory findings for all patients with a negative swab from admission until discharge. Cases were evaluated using the Public Health England (PHE) testing criteria for possible COVID cases.

We defined swab-negative clinical COVID-19 cases as follows: (a) clinical COVID-19 or high level of suspicion as defined by the treating medical team (as recorded in the medical notes, discharge documents or death certificate); and (b) RT-PCR swab-negative (on initial and any subsequent tests performed). Patients who tested positive on subsequent swabs while in hospital were not included in the reported cohort.

The swab-negative clinical COVID-19 group of patients was compared to a subgroup of a previously described swab-positive cohort who were similarly admitted via general medical admissions ${ }^{12}$. Chi-square, Fisher's and rank sum tests were used, as appropriate, to compare the cohorts' characteristics, and odds ratios (OR) calculated to assess differences in the outcomes of respiratory deterioration, defined as requiring greater than $60 \%$ oxygen, and death. Lastly, we assessed the cumulative risk of the competing outcomes of hospital discharge and death over time using the using the Nelson-Aelen estimator.

The study was approved by the ICHNT clinical governance team. As we report on routinely collected nonidentifiable clinical audit data, no individual informed consent was required under the UK policy framework for health and social care. All methods were conducted in accordance with relevant guidelines and regulations.

\section{Results}

We identified 1,119 emergency medical admissions with initial clinical suspicion of COVID-19 who had a SARSCoV-2 swab performed (Fig. 1). Initial swab was negative in $456(41 \%)$ and positive in 663 (59\%) patients. 62\% $(281 / 456)$ of those who were swab-negative received an alternative diagnosis. 47 of the swab-negative cohort later tested positive for SARS-CoV-2 on repeat PCR testing and were not included within the clinical COVID19 cohort. Patients who tested positive on subsequent testing had between 2 and 4 nasopharyngeal swabs in total with a median number of 2 . The most common alternative diagnoses included community acquired pneumonia, lower respiratory tract infection and exacerbations of chronic lung disease such as chronic obstructive pulmonary disease.

Overall, 20\% (94/456) of swab-negative cases were identified as swab-negative clinical COVID-19. 98\% (92/94) of these cases fulfilled PHE criteria for patients eligible to be swabbed for SARS-CoV-2 compared to 
$84 \%$ of the entire swab-negative group. $41 / 94$ had repeat swab testing performed and remained negative. Those who had repeat swabbing had between 2 and 3 swabs in total with a median number of 2 .

The demographic profile and symptomatology of the swab-positive and swab-negative cohorts were similar, with high rates of influenza-like symptoms, cough and fever in both groups (Table 1). Typical features such as shortness of breath was higher in the swab-negative clinical COVID-19 cohort (75/94 (79.79\%) vs $312 / 478(66.67 \%) p=0.017)$. The swab-negative cohort was more likely to have chest radiographs reporting typical COVID-19 appearances than the swab-positive cohort (53/77 (68.83\%) vs 195/389 (50.13\%); $p=0.004)$. Only 18 patients within the swab-negative clinical COVID-19 cohort had a CT chest performed during their admission. 15/18 CT chests were reported as being consistent with COVID-19 or had COVID-19 within the reported differential.

Overall haematological and biochemical findings that have been associated with COVID-19 including raised CRP, d-dimer and ferritin were similarly distributed between the two cohorts. A higher proportion of patients had moderate lymphopenia within the swab-negative group (65/94 (69.15\%) vs 258/460 (56.09\%); $p=0.026)$.

Patients in the swab-negative group presented slightly later in their clinical course after symptom onset, at a median of 7 days (IQR 4.3-13) for the RT-PCR-negatives compared to 6 days (IQR 3-10) within the RT-PCRpositives cohort $(p<0.001)$. Interestingly, RT-PCR-negative patients had better hospitalisation outcomes than RT-PCR-positives. The former had a $57 \%$ lower probability of requiring $\geq 60 \%$ supplementary oxygen during hospitalisation (OR $0.43,95 \%$ CI $0.26-0.70, p<0.001$ ), a shorter length of hospital stay (median 5 days vs 6 days, $p<0.001$ ) and $60 \%$ lower probability of death (OR $0.40,95 \%$ CI $0.22-0.72, p<0.001$ ). The cumulative risk of being discharged alive was significantly higher in the swab-negative group than the positive group $(p<0.001)$. Furthermore, the swab-negative cohort have a lower cumulative risk of in-hospital mortality than positives $(p<0.001)$ (Fig. 2).

\section{Discussion}

We find one in five symptomatic patients admitted to the medical department who had a negative SARS-CoV-2 swab received a clinical diagnosis of COVID-19 despite negative testing. This represents a 13\% increase in the total number of hospitalized COVID-19 patients over the study period compared to considering only swab positive patients. Overall the clinical, biochemical and radiological features of the swab-negative clinical COVID-19 cohort did not differ significantly to the swab-positive cohort. Typical features of COVID-19 such as shortness of breath, typical chest $\mathrm{x}$-ray findings and lymphopenia are more common in the swab-negative cohort. The likely explanation is that swab-positive patients with a range of symptoms are classified as COVID-19 patients based on the positive swab test, whilst swab-negative patients diagnosed as having COVID-19 have their diagnosis based on typical symptoms. Swab-negative patients tended to have a longer delay between symptom onset and hospitalization, and had significantly better hospitalization outcomes, including mortality. Whether this potentially less aggressive phenotype is related to a lower viral load, variations in immune response or a different stage of illness presenting after period of peak viral replication is not yet known. Meta-analysis of studies reporting viral load and shedding of SARS-CoV-2 report that upper respiratory tract viral titres peak in the first week of illness ${ }^{13}$. Another analysis of published studies have reported the probability of false negative swab SARS-CoV-2 swab testing increases after day 3 of symptoms and upper respiratory tract SARS-CoV-2 viral load reduces over time $^{9,14}$. Further research including immunological profiling would help further elucidate underlying disease mechanisms.

Our results provide evidence to validate swab-negative COVID-19 as a real clinical entity commonly encountered in hospital settings, findings which have important implications for current clinical practice and public health guidance ${ }^{15}$. This is supported by reported cohorts in the literature of patients with clinical features of COVID-19 with negative RT-PCR swab testing and positive serological testing ${ }^{16-18}$.

Our study highlights that clinicians caring for COVID-19 patients should maintain a high clinical suspicion, even in the presence of a negative swab result. Our results suggest that a combination of clinical, radiological and biochemical features in keeping with COVID-19 disease, rather than swab results alone, should guide clinical management.

Furthermore, managing swab-negative clinical COVID-19 within hospital poses disease control challenges. Cohorting this group with swab-positive patients presents potential risk of SARS-CoV-2 infection, if the clinical diagnosis is incorrect. Alternatively, isolating these patients from both swab-negative low-risk patients and swabpositive groups would place significant strain on hospital resources and may be unfeasible during an outbreak. Nasopharyngeal swabbing is vulnerable to inter-operator variation and poor sampling technique may explain why patients with high clinical suspicion of COVID-19 had negative swab testing. Repeated testing may be beneficial in some cases however in our cohort 41/94 swab negative clinical COVID-19 cases had a repeat swab which remained negative. Testing on sputum or bronchial lavage may provide greater sensitivity ${ }^{6-8}$. However, their use is limited by feasibility and potential risk to healthcare workers. Serological testing to confirm exposure to SARS-CoV-2 is likely the optimal second-line test as it is not operator-dependent, is low-risk to healthcare workers, and can feasibly be performed on a large number of patients. Serological proof of exposure could reduce concern regarding cohorting with swab-positive patients. Although serological testing has demonstrated good sensitivity and specificity performance can vary between serological kits ${ }^{19}$.

Lastly, but importantly, from a public health perspective, swab-negative clinically diagnosed COVID-19 patients may not be accurately captured in surveillance statistics, thereby underestimating healthcare demand. As the pandemic evolves countries with reducing incidence should consider this clinical group and how they should be addressed when planning interventions to manage second-wave outbreaks and contact tracing.

Our study is limited by its retrospective nature and likely under-estimates the proportion of patients with swab-negative clinical COVID-19, as it requires a high degree of clinical confidence without alternative diagnosis. 


\begin{tabular}{|c|c|c|c|}
\hline & $\begin{array}{l}\text { RT-PCR-positive } \\
(\mathrm{n}=468)\end{array}$ & $\begin{array}{l}\text { RT-PCR-negative } \\
(\mathrm{n}=94)\end{array}$ & $p$ value \\
\hline \multicolumn{4}{|l|}{ Demography } \\
\hline Male, n (\%) & $288(61.5 \%)$ & $57(60.6 \%)$ & $0.962 *$ \\
\hline Median age (IQR) & $68(54-79)$ & $67(54.25-78)$ & $0.638^{\dagger}$ \\
\hline \multicolumn{4}{|l|}{ Symptoms } \\
\hline Influenza-like syndrome, n (\%) & $341(72.9 \%)$ & $74(78.7 \%)$ & 0.293 * \\
\hline Cough, n (\%) & $353(75.4 \%)$ & $71(75.5 \%)$ & 1.000 * \\
\hline Fever, n (\%) & $389(83.1 \%)$ & $75(79.8 \%)$ & $0.530^{*}$ \\
\hline Nasal discharge, $\mathrm{n}(\%)$ & $9(1.9 \%)$ & $3(3.2 \%)$ & $0.433^{*}$ \\
\hline Shortness of breath, $\mathrm{n}(\%)$ & $312(66.7 \%)$ & $75(79.8 \%)$ & $0.017^{*}$ \\
\hline Wheezing, n (\%) & $1(0.2 \%)$ & $4(4.3 \%)$ & $0.003^{*}$ \\
\hline Anosmia, n (\%) & $12(2.6 \%)$ & $10(10.6 \%)$ & 0.001 * \\
\hline \multicolumn{4}{|l|}{ Admission oxygen requirements $\left(\mathrm{FiO}_{2}\right)$} \\
\hline Room air, n (\%) & $254(54.3 \%)$ & $50(53.2 \%)$ & $0.937^{*}$ \\
\hline 24 to $<40 \%$ & $108(23.1 \%)$ & $31(33.0 \%)$ & $0.058^{*}$ \\
\hline 40 to $<60 \%$ & $8(1.7 \%)$ & $3(3.2 \%)$ & $0.405^{*}$ \\
\hline$>60 \%$ & $98(20.9 \%)$ & $10(10.6 \%)$ & 0.030 * \\
\hline \multicolumn{4}{|l|}{ Chest radiograph } \\
\hline Normal CXR & $65 / 389(16.7 \%)$ & $7 / 77(9.1 \%)$ & $0.129^{*}$ \\
\hline Typical COVID-19 CXR & $195 / 389(50.2 \%)$ & $53 / 77(68.8 \%)$ & $0.004^{*}$ \\
\hline Atypical COVID-19 CXR & $106 / 389(27.3 \%)$ & $15 / 77(19.5 \%)$ & 0.201 * \\
\hline Non-COVID abnormal CXR & $23 / 389(5.9 \%)$ & $2 / 77(2.6 \%)$ & $0.403^{*}$ \\
\hline \multicolumn{4}{|l|}{ Laboratory test } \\
\hline Lymphocytes $\geq 1.1$ & $154 / 460(33.5 \%)$ & $25(26.6 \%)$ & $0.238^{*}$ \\
\hline Lymphocytes $0.5-1.0$ & $258 / 460(56.1 \%)$ & $65(69.2 \%)$ & $0.026^{*}$ \\
\hline Lymphocytes $<0.5$ & $43 / 460(9.4 \%)$ & $4(4.3 \%)$ & $0.152^{*}$ \\
\hline $\mathrm{CRP} \geq 100$ & $230 / 448(51.3 \%)$ & $55 / 93(59.1 \%)$ & $0.209^{*}$ \\
\hline CRP 10-99 & $186 / 448(41.5 \%)$ & $33 / 93(35.5 \%)$ & $0.336^{*}$ \\
\hline $\mathrm{CRP}<10$ & $32 / 448(7.1 \%)$ & $5 / 93(5.4 \%)$ & $0.698^{*}$ \\
\hline D-dimer $\geq 3000$ & $42 / 225(18.7 \%)$ & $16 / 66(24.2 \%)$ & 0.411 * \\
\hline D-dimer 2000-2999 & $29 / 225(12.9 \%)$ & $9 / 66(13.6 \%)$ & $1.000^{*}$ \\
\hline D-dimer 1000-1999 & $64 / 225(28.4 \%)$ & $19 / 66(28.8 \%)$ & 1.000 * \\
\hline D-dimer 500-999 & $63 / 225(28.0 \%)$ & $18 / 66(27.3 \%)$ & $1.000 *$ \\
\hline $\mathrm{D}$-dimer $<500$ & $27 / 225(12.0 \%)$ & $4 / 66(6.1 \%)$ & $0.255^{*}$ \\
\hline $\mathrm{LDH} \geq 243$ & $166 / 179(92.7 \%)$ & $48 / 51(94.1 \%)$ & $0.976^{*}$ \\
\hline $\mathrm{LDH}<243$ & $13 / 179(7.3 \%)$ & $3 / 51(5.9 \%)$ & $1.000^{*}$ \\
\hline $\mathrm{CK} \geq 320$ & $63 / 210(30.0 \%)$ & $16 / 64(25.0 \%)$ & $0.538^{*}$ \\
\hline $\mathrm{CK}<320$ & $147 / 210(70.0 \%)$ & $48 / 64(75.0 \%)$ & $0.538^{*}$ \\
\hline Ferritin $\geq 5000$ & $13 / 259(5.0 \%)$ & $4 / 68(5.9 \%)$ & $0.761^{*}$ \\
\hline Ferritin 1000-4999 & $94 / 259(36.3 \%)$ & $27 / 68(39.7 \%)$ & $0.706^{*}$ \\
\hline Ferritin 500-999 & $73 / 259(28.2 \%)$ & $15 / 68(22.1 \%)$ & $0.390^{*}$ \\
\hline Ferritin 300-499 & $38 / 259(14.7 \%)$ & $10 / 68(14.7 \%)$ & $1.000^{*}$ \\
\hline Ferritin $<300$ & $41 / 259(15.8 \%)$ & $12 / 68(17.7 \%)$ & 0.860 * \\
\hline \multicolumn{4}{|l|}{ Clinical course } \\
\hline Median (IQR) days prior to admission & $6(3-10)$ & $7(4.25-13)$ & $0.013^{\dagger}$ \\
\hline Median (IQR) length of stay & $6(4-10)$ & $5(2.25-6)$ & $<0.001^{\dagger}$ \\
\hline Received $\geq 60 \% \mathrm{FiO}_{2}, \mathrm{n}(\%)$ & $215(45.9 \%)$ & $25(26.6 \%)$ & 0.001 * \\
\hline Died, n (\%) & $151(32.3 \%)$ & $15(16.0 \%)$ & 0.002 * \\
\hline
\end{tabular}

Table 1. Description of clinical characteristics and clinical course. CK, creatine kinase; CRP, C-reactive protein; EWS, early warning score; $\mathrm{FiO}_{2}$, inspiratory fraction of oxygen; IQR, interquartile range; $\mathrm{LDH}$, lactate dehydrogenase. ${ }^{\star}$ Pearson's Chi-squared test with Yates' continuity correction. ${ }^{\dagger}$ Wilcoxon rank sum test with continuity correction. ${ }^{\ddagger}$ Fisher's exact test for count data. 


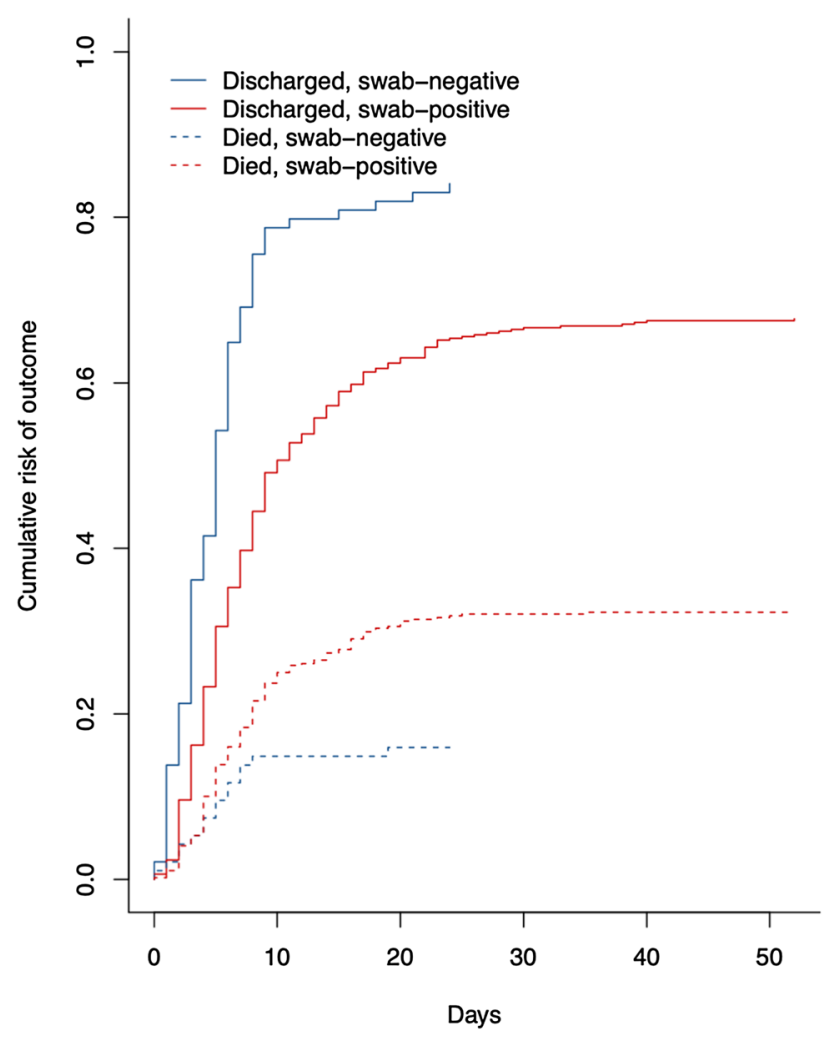

Figure 2. Cumulative risk of hospitalization outcomes by swab status.

Our relatively small cohort size is therefore a further limitation. The lack of positive confirmatory test means we cannot be sure of the true diagnosis in the clinically diagnosed swab negative group. However, this does not detract from the clinical problems this group presents to hospital management and epidemiological assessment. In the absence of improved testing or routine second line testing for patients who have high clinical suspicion of COVID-19 but negative swab testing, this group is likely to continue to pose a clinical challenge. During the period of data collection changes in local testing policy meant that not all patients were retested for SARS-CoV-2 which could limit the generalizability of some of our results. However this is probably more reflective of the realworld context, particularly where resources are limited. Lastly, it is also possible that, given our relatively small cohort size, demographic and clinical differences between swab-positive and swab-negative patients were not evident. This finding warrants further investigation, as larger studies or meta-analyses are needed to allow the investigation of potential differences between such cohorts and even undertake multivariable regression analyses.

Patients with strong clinical features of COVID-19 who have negative nasopharyngeal RT-PCR test results are a common but understudied clinical group. We found they do not differ significantly to similar swab-positive patients but seem to have better outcomes. Healthcare services should recognize and plan for the management of this group when making disease control interventions and epidemiological assessments.

Received: 22 September 2020; Accepted: 5 January 2021

Published online: 28 January 2021

\section{References}

1. World Health Organization. Coronavirus disease (COVID-2019) situation reports. 2020. https://www.who.int/emergencies/disea ses/novel-coronavirus-2019/situation-reports (accessed Dec 2020).

2. World Health Organization. Global surveillance for COVID-19 caused by human infection with COVID-19 virus: interim guidance. 2020. https://www.who.int/publications-detail/global-surveillance-for-covid-19-caused-by-human-infection-with-covid -19-virus-interim-guidance (accessed 18 May 2020).

3. World Health Organization. WHO COVID-19 Case definition. 2020. https://www.who.int/publications/i/item/WHO-2019-nCoVSurveillance_Case_Definition-2020.1. (accessed Dec 2020).

4. UK Government. Number of coronavirus (COVID-19) cases and risk in the UK. Number of coronavirus (COVID-19) cases and risk in the UK. 2020. https://www.gov.uk/guidance/coronavirus-covid-19-information-for-the-public (accessed 27 Apr 2020).

5. Ai T, Yang Z, Hou H, et al. Correlation of Chest CT and RT-PCR Testing in Coronavirus Disease 2019 (COVID-19) in China: A Report of 1014 Cases. Radiology 2020;:200642. https://doi.org/10.1148/radiol.2020200642.

6. Hase, R. et al. A case of imported COVID-19 diagnosed by PCR-positive lower respiratory specimen but with PCR-negative throat swabs. Infect. Dis. 52, 423-426. https://doi.org/10.1080/23744235.2020.1744711 (2020).

7. Winichakoon, P. et al. Negative nasopharyngeal and oropharyngeal Swabs do not rule out COVID-19. J. Clin. Microbiol. 58, e00297-e320. https://doi.org/10.1128/JCM.00297-20 (2020).

8. Han, H. et al. SARS-CoV-2 RNA more readily detected in induced sputum than in throat swabs of convalescent COVID-19 patients. Lancet Infect. Dis. https://doi.org/10.1016/S1473-3099(20)30174-2 (2020). 
9. Kucirka, L. M. et al. Variation in false-negative rate of reverse transcriptase polymerase chain reaction-based SARS-CoV-2 tests by time since exposure. Ann. Int. Med. https://doi.org/10.7326/M20-1495 (2020).

10. Kulkarni, S. et al. Repurposed immunomodulatory drugs for Covid-19 in pre-ICu patients-mulTi-Arm Therapeutic study in preICu patients admitted with Covid-19-repurposed drugs (TACTIC-R): a structured summary of a study protocol for a randomised controlled trial. Trials 21, 626. https://doi.org/10.1186/s13063-020-04535-4 (2020).

11. Horby, P. et al. Effect of dexamethasone in hospitalized patients with COVID-19: preliminary report. Infect. Dis. (Except HIV/ AIDS) https://doi.org/10.1101/2020.06.22.20137273 (2020).

12. Perez-Guzman, P. N. et al. Clinical characteristics and predictors of outcomes of hospitalized patients with COVID-19 in a multiethnic London NHS Trust: a retrospective cohort study. Clin. Infect. Dis. https://doi.org/10.1093/cid/ciaa1091 (2020).

13. Cevik, M. et al. SARS-CoV-2, SARS-CoV, and MERS-CoV viral load dynamics, duarion of viral shedding and infectiousness: a systematic review and meta-analysis. Lancet Microbe https://doi.org/10.1016/S2666-5247(20)30172-5 (2020).

14. Walsh KA, Jordan K, Clyne B, et al. SARS-CoV-2 detection, viral load and infectivity over the course of an infection. J. Infect. (2020).

15. Public Health England. COVID-19: investigation and initial clinical management of possible cases. 2020. https://www.gov.uk/gover $\mathrm{nment} /$ publications/wuhan-novel-coronavirus-initial-investigation-of-possible-cases/investigation-and-initial-clinical-manag ement-of-possible-cases-of-wuhan-novel-coronavirus-wn-cov-infection\#criteria. (accessed 18 May 2020).

16. Rong, Y. et al. Clinical characteristics and risk factors of mild-to-moderate COVID-19 patients with false-negative SARS-CoV-2 nucleic acid. J. Med. Virol. https://doi.org/10.1002/jmv.26242 (2020).

17. Di Paolo, M. et al. False-negative RT-PCR in SARS-CoV-2 disease: experience from an Italian COVID-19 unit. ERJ Open Res. 6 , 00324-02020. https://doi.org/10.1183/23120541.00324-2020 (2020).

18. López de la Iglesia, J. et al. Predictive factors of COVID-19 in patients with negative RT-qPCR. Medicina de Familia SEMERGEN 46, 6-11. https://doi.org/10.1016/j.semerg.2020.06.010 (2020).

19. Coste, A. T. et al. Comparison of SARS-CoV-2 serological tests with different antigen targets. Infect. Dis. (Except HIV/AIDS) https ://doi.org/10.1101/2020.07.09.20149864 (2020).

\section{Author contributions}

S.N.-Conceived study, data interpretation and provided clinical expertise and critical revision of the manuscript for intellectual and scientific content. P.M.-Conceived study, wrote the first manuscript and led on acquisition of data, interpreted data, provided clinical expertise and critical revision of the manuscript for intellectual and scientific content. P.P.-Conceived study, performed statistical analysis, interpreted data, provided clinical expertise and critical revision of the manuscript for intellectual and scientific content. A.C.- Supported data acquisition and curation and critical revision of the manuscript for intellectual and scientific content. N.K.Supported data acquisition and curation and critical revision of the manuscript for intellectual and scientific content A.D.- Supported data acquisition and curation and critical revision of the manuscript for intellectual and scientific content. S.M.-Supported data acquisition and curation and critical revision of the manuscript for intellectual and scientific content. M.D.K.-Performed statistical analysis, data interpretation and critical revision of the manuscript for intellectual and scientific content. G.C.-Provided clinical expertise and critical revision of the manuscript for intellectual and scientific content. M.R.T.-Provided clinical expertise and scientific expertise to lead the discussion of clinical and public health implications and critical revision of the manuscript for intellectual and scientific content. P.J.W.- Provided scientific expertise to lead the discussion of clinical and public health implications and critical revision of the manuscript for intellectual and scientific content. T.B.H.-Provided scientific expertise to lead the discussion of clinical and public health implications and critical revision of the manuscript for intellectual and scientific content. K.H.-Provided scientific expertise to lead the discussion of clinical and public health implications and critical revision of the manuscript for intellectual and scientific content.

\section{Funding}

This work was supported by Medical Research Council Centre for Global Infectious Disease Analysis and the NIHR HPRU in Modelling and Health Economics. SN, TBH, KH, PJW and PP were supported by the MRC Centre for Global Infectious Disease Analysis (MR/R015600/1); this award is jointly funded by the UK Medical Research Council (MRC) and the UK Foreign, Commonwealth \& Development Office (FCDO) under the $\mathrm{MRC/FCDO}$ Concordat agreement and is also part of the EDCTP2 program supported by the European Union (EU). PJW is supported by the NIHR HPRU in Modelling and Health Economics, a partnership between Public Health England, Imperial College London and LSHTM (grant code NIHR200908). The authors would also like to acknowledge the support of the Imperial BRC Centre and J-IDEA. The authors would like to thank all patients and staff at Imperial College Healthcare NHS Trust. Role of the Funder/Sponsor: The funding organizations had no role in the design and conduct of the study; collection, management, analysis, and interpretation of the data; preparation, review, or approval of the manuscript; and decision to submit the manuscript for publication. Disclaimer: The views expressed are those of the authors and not necessarily those of the United Kingdom (UK) Department of Health and Social Care, EU, FCDO, MRC, National Health Service, NIHR, or PHE.

\section{Competing interests}

The authors declare no competing interests.

\section{Additional information}

Correspondence and requests for materials should be addressed to S.N.

Reprints and permissions information is available at www.nature.com/reprints.

Publisher's note Springer Nature remains neutral with regard to jurisdictional claims in published maps and institutional affiliations. 
(c) (i) Open Access This article is licensed under a Creative Commons Attribution 4.0 International cc) License, which permits use, sharing, adaptation, distribution and reproduction in any medium or format, as long as you give appropriate credit to the original author(s) and the source, provide a link to the Creative Commons licence, and indicate if changes were made. The images or other third party material in this article are included in the article's Creative Commons licence, unless indicated otherwise in a credit line to the material. If material is not included in the article's Creative Commons licence and your intended use is not permitted by statutory regulation or exceeds the permitted use, you will need to obtain permission directly from the copyright holder. To view a copy of this licence, visit http://creativecommons.org/licenses/by/4.0/.

(C) The Author(s) 2021 